\title{
Touch-screen-guided task reveals a prosocial choice tendency by chimpanzees (Pan troglodytes)
}

\author{
Renata S Mendonça ${ }^{\text {Corresp., }}{ }^{1,2}{ }^{\text {, }}$ Christoph D Dahl ${ }^{3}$, Susana Carvalho ${ }^{2,4,5,6}$, Tetsuro Matsuzawa ${ }^{7}$, Ikuma Adachi ${ }^{8}$ \\ 1 Primate Research Institute, Section of Language and Intelligence, Kyoto University, Inuyama, Japan \\ ${ }^{2}$ Centre for Functional Ecology - Science for People \& the Planet, Department of Life Sciences, University of Coimbra, Coimbra, Portugal \\ 3 Institute of Biology, Department of Comparative Cognition, University of Neuchatel, Neuchâtel, NE, Switzerland \\ 4 Primate Models for Behavioural Evolution Lab, Institute of Cognitive and Evolutionary Anthropology, University of Oxford, Oxford, United Kingdom \\ 5 Interdisciplinary Centre for Archaeology and Evolution of Human Behaviour (ICArEHB), University of Algarve, Faro, Portugal \\ 6 Gorongosa Restoration Project, Gorongosa National Park, Sofala, Mozambique \\ 7 Institute for Advanced Studies, University of Kyoto, Kyoto, Japan \\ 8 Primate Research Institute, Center for International Collaboration and Advanced Studies in Primatology, Kyoto University, Inuyama, Japan \\ Corresponding Author: Renata S Mendonça \\ Email address: mendonca.renata.8m@kyoto-u.ac.jp
}

Humans help others even without direct benefit for themselves. However, the nature of altruistic (i.e. only the other benefits) and prosocial (i.e. self and other both benefit) behaviors in our closest living relative, the chimpanzee, remains controversial. To address this further, we developed a touch-screen-guided task that allowed us to increase the number of trials for a thorough test of chimpanzees' prosocial and altruistic tendencies. Mother-offspring dyads were tested in the same compartment; one was the actor while the other was the recipient. In experiment 1, the actor chose among three options: prosocial, selfish (only the actor benefited) and altruistic. To better understand the nature of the chimpanzees' choices and to improve experimental control, we conducted two additional experiments. Experiment 2 consisted of two-option choices interspersed with three-option choices, and in experiment 3 the two-option choice were blocked across all trials. The results of experiment 1 clearly showed that chimpanzees acted prosocially in the touchscreen-guided task, choosing the prosocial option on an average of $79 \%$ of choices. Five out of the six chimpanzees showed the preference to act prosocially against chance level. The preference for the prosocial option persisted when conditions were changed in experiments 2 and 3 . When only selfish and altruistic options were available in experiments 2 and 3, chimpanzees preferred the selfish option. These results suggest that 1) most individuals understood the nature of the task and modified their behavior according to the available options, 2) five out of the six chimpanzees chose to act prosocially when they had the option to, and 3) offspring counterbalanced between altruistic and selfish, when given those two options perhaps to avoid suffering repercussions from the mother. 
1 Touch-screen-guided task reveals a prosocial choice tendency by chimpanzees (Pan

\section{2 troglodytes)}

3 Renata S. Mendonça ${ }^{1,2} \uparrow *$, Christoph D. Dahl ${ }^{3} \dagger$, Susana Carvalho ${ }^{2,4,5,6}$, Tetsuro Matsuzawa ${ }^{1,7}$, 4 Ikuma Adachi ${ }^{8}$

$5 \quad{ }^{1}$ Primate Research Institute, Kyoto University, Section Language and Intelligence, 41-2 Kanrin,

6 Inuyama, Aichi, 484-8506, Japan

$7 \quad{ }^{2}$ Centre for Functional Ecology - Science for People \& the Planet, Department of Life Sciences,

8 University of Coimbra, Calçada Martim de Freitas, 3000-456 Coimbra, Portugal

$9{ }^{3}$ Institute of Biology, University of Neuchatel, Department of Comparative Cognition, Rue

10 Emile-Argand 112000 Neuchâtel, Switzerland

$11{ }^{4}$ Primate Models for Behavioural Evolution Lab, Institute of Cognitive and Evolutionary

12 Anthropology, University of Oxford, OX2 6PN, United Kingdom

$13{ }^{5}$ Interdisciplinary Centre for Archaeology and Evolution of Human Behaviour (ICArEHB),

14 University of Algarve, Gambelas, Faro, Portugal

$15 \quad{ }^{6}$ Gorongosa National Park, Sofala, Mozambique

$16{ }^{7}$ Institute for Advanced Studies, Kyoto University, Yoshida Ushinomiya-cho, Sakyo Kyoto, 606-

17 8501, Japan

$18{ }^{8}$ Primate Research Institute, Kyoto University, Center for International Collaboration and

19 Advanced Studies in Primatology, 41-2 Kanrin, Inuyama, Aichi, 484-8506, Japan

*Correspondence to: Renata S. Mendonça (renatadasilva.mendonca@gmail.com) 
$21 \uparrow$ These authors contributed equally.

22 Abstract

23 Humans help others even without direct benefit for themselves. However, the nature of altruistic

24 (i.e. only the other benefits) and prosocial (i.e. self and other both benefit) behaviors in our closest

25 living relative, the chimpanzee, remains controversial. To address this further, we developed a

26 touch-screen-guided task that allowed us to increase the number of trials for a thorough test of

27 chimpanzees' prosocial and altruistic tendencies. Mother-offspring dyads were tested in the same

28 compartment; one was the actor while the other was the recipient. In experiment 1, the actor chose

29 among three options: prosocial, selfish (only the actor benefited) and altruistic. To better

30 understand the nature of the chimpanzees' choices and to improve experimental control, we

31 conducted two additional experiments. Experiment 2 consisted of two-option choices interspersed

32 with three-option choices, and in experiment 3 the two-option choice were blocked across all trials.

33 The results of experiment 1 clearly showed that chimpanzees acted prosocially in the touch-screen-

34 guided task, choosing the prosocial option on an average of $79 \%$ of choices. Five out of the six

35 chimpanzees showed the preference to act prosocially against chance level. The preference for the

prosocial option persisted when conditions were changed in experiments 2 and 3 . When only

37 selfish and altruistic options were available in experiments 2 and 3, chimpanzees preferred the selfish option. These results suggest that 1) most individuals understood the nature of the task and modified their behavior according to the available options, 2) five out of the six chimpanzees chose to act prosocially when they had the option to, and 3) offspring counterbalanced between altruistic and selfish, when given those two options perhaps to avoid suffering repercussions from the 42 mother. 
45

46

47

48

Introduction

Humans are clearly a case in which social exchange increases the relative fitness of individuals who engage in altruistic behaviors, enabling altruism to diffuse through subsequent generations (Fehr \& Fischbacher, 2003; Warneken \& Tomasello, 2009). Humans frequently help others without directly benefiting themselves (Fehr \& Gächter, 2002; Fehr \& Fischbacher, 2004). Prosocial behavior is described as any behavior that includes actions intended to benefit another, such as helping, comforting, sharing resources and cooperating (Batson \& Powell, 2003). Altruism is a motivational concept in which the actor does not consciously regard his self-interests (Hoffman, 1978; Batson \& Powell, 2003). Therefore, this behavior can benefit the recipient while entailing costs to the actor, or in the absence of any obvious proximate reward (Batson \& Powell, 2003; de Waal, 2008). This concept contrasts with egoism (here referred to as selfish behavior), which has the ultimate goal of increasing one's own welfare (Mueller, 1986). How did prosocial behaviors evolve in humans? Comparative studies can provide important perspectives for addressing this question. In recent decades multiple studies have explored prosocial and altruistic behaviors in nonhuman primate species (de Waal et al., 2008; Lakshminarayanan \& Santos, 2008; Cronin, Schroeder \& Snowdon, 2010; Skerry, Sheskin \& Santos, 2011; Horner et al., 2011a; Takimoto \& Fujita, 2011; Suchak \& de Waal, 2012; Kim et al., 2015). To understand the mechanisms that underlie prosocial and altruistic behavior, the chimpanzee is a good model for the following three major reasons: 1) they share a recent common ancestry with humans, which makes them a good comparative model for studying the evolution of human behavior (McGrew, 2010); 2) some observational studies have reported prosocial behavior in chimpanzees (Nishida \& Hosaka, 1996; Watts, 1998; Langergraber, Mitani \& Vigilant, 2007; Crockford et al., 2012); and 
67 3) empirical evidence shows that chimpanzees understand other individuals' intentions (Hare, Call

68 \& Tomasello, 2001, Yamamoto, Humle \& Tanaka, 2012). (Hirata, 2009). However, the issue of prosociality remains controversial, as some studies have failed to show such tendencies (Silk et al., 2005; Jensen et al., 2006; Vonk et al., 2008) and other reported prosocial tendencies only slightly above chance level (Warneken et al., 2007; Horner et al., 2011a; Melis, Schneider \& Tomasello, 2011; Melis et al., 2011; Claidière et al., 2015). Two main experimental paradigms have been used to test prosociality in non-human primates (Horner et al., 2011a), namely using: 1) giving assistance tests, in which the subject has to choose between helping, by providing instrumental help, or not helping the recipient and 2) prosocial choices tests (PCT), in which the subject has to choose between a prosocial (allowing subject and recipient to be rewarded) or selfish option (only the subject is rewarded). Some PCT studies have failed to show a clear prosocial preferences in chimpanzees (Silk et al., 2005; Jensen et al., 2006), arguably due to methodological constraints. Even with improved paradigms results are unclear $(60 \%$ prosocial) (Horner et al., 2011a) and open to challenge (Skoyles, 2011), given the frequent selection (40\%) of selfish tokens, when a choice between selfish and prosocial tokens was presented by the experimenter. However, authors have argued that organisms do not choose categorically but rather sample the choices from time to time, which may result in a high proportion of selfish choices (Horner et al., 2011b).

We developed a touch-screen-guided task to re-examine the existence of prosocial and altruistic behaviors, as well as the factors modulating their choices, using a new paradigm. We tested three mother-offspring pairs of chimpanzees, who had experience with various computercontrolled experiments (Matsuzawa, 2003; Matsuzawa, 2006; Martin et al., 2014). Unlike most of 
90 the prosociality studies (but see: House et al.; 2014; Suchak et al., 2014; Claidière et al., 2015), we

91 tested the actor and recipient individuals in the same compartment and we used a touch-screen-

92 guided procedure that allowed us to increase the number of trials per individual. A prosocial option

93 was defined as the chimpanzee playing the role of actor choosing to reward both actor and

94 recipient. An altruistic option was defined as the act of providing reward only to the recipient. A

95 selfish option was defined as the actor choosing to reward only himself. We ran three experiments

96 to examine how prosocial, selfish and altruistic tendencies were modulated across different

97 conditions. In experiment 1 , chimpanzees were requested to choose among prosocial (P), selfish

98 (S) and altruistic (A) options. In the experiment 2 and 3 they were given two of the three options.

99 Experiment 2 consisted of choosing between two out of three choices that were presented

100 randomly across the trials. Experiment 3 consisted of three sessions, each one with two out three

101 choices (for example, one session only with prosocial and altruistic options, another with altruistic

102 and selfish, and another with prosocial and selfish) blocked across the trials.

We hypothesized that chimpanzees show a tendency to behave prosocially (above selfishly

104

105

106

107

108

109

110

111

112 and altruistically), and this tendency varies according different conditions. The following predictions were formulated for each condition/experiment: experiment 1) if chimpanzees have a tendency to behave prosocial, they should choose the prosocial option more often than the selfish and altruistic options; experiment 2) if chimpanzees understand the meaning of the outcomes a) they should keep their prosocial preference and b) when given a choice between two out of the three options, they should show a preference for one of the options; experiment 3) if chimpanzees are presented with two out of the three options constantly across the trials, they may counterbalance their choices to avoid repercussions from other individuals. 


\section{General Methods}

\section{Participants}

115 Six chimpanzees (Pan troglodytes): one juvenile male (Ayumu, 12 years old), two juvenile

116 females (Cleo and Pal, around 12 years of age) and three adult females (Ai, Chloe, and Pan, all

117 around 30 years of age) participated as mother-offspring pairs. Because of their mother-offspring

118 relationship individuals had to be tested in the same compartment: Ai with Ayumu (Am), Chloe

119 (Ch) with Cleo (Cl), Pan (Pn) with Pal (Pl) (Fig. 1). The chimpanzees live in groups of six and 120 seven individuals in indoor-outdoor enclosures at the Primate Research Institute, Kyoto 121 University. The outdoor enclosure $\left(770 \mathrm{~m}^{2}\right)$ is environmentally enriched with artificial streams 122 containing fish and more than 400 species of plants, in addition to ropes and climbing structures 123 up to $15 \mathrm{~m}$ high, and has direct access to indoor quarters. All subjects had previously participated 124 in various computer-controlled perceptual-cognitive experiments (Matsuzawa, 2003; Matsuzawa, 125 2006; Adachi, 2014) including some in similar social settings (Martin, Biro \& Matsuzawa, 2011; 126 Martin et al., 2014).

129 We used two 17-inch LCD touch panel displays (1280 x 1024 pixels) controlled by customwritten software under Visual Basic 2010 (Microsoft Corporation, Redmond, Washington, USA). 131 Chimpanzees sat in one experimental chamber (approximately $2.5 \mathrm{~m}$ wide, $2.5 \mathrm{~m}$ deep, $2.1 \mathrm{~m}$ 132 high), while the experimenters sat outside the booth, separated from the chimpanzees by 133 transparent acrylic panels (Fig. 1). The displays were placed into the acrylic panels. The 134 appropriate distance between the active subject and its display was 40 to $50 \mathrm{~cm}$. Options appeared 
135 on the screen in sizes of about 3 to 4 degrees of visual angle. The subjects responded by touching

136 the options on the display surface with a finger. A transparent acrylic panel fitted with an opening

137 allowed manual contact with the display while protecting it from damage. A food tray was installed

138 below each display, for delivering food rewards via a universal feeder (Bio Medica BUF-310P50).

139 Displays and feeders were automatically controlled by the same program that controlled the 140 display of the stimuli.

142 Stimuli

143 To initiate the task, a circular button was presented as stimuli in the bottom of the actor's screen. After pressing the start key, three grayscale 3-D shape options (cube, cylinder and sphere) horizontally aligned with equal spacing on the computer monitor of one of the two chimpanzees

146 (Fig. 2). Each symbol represented each given option: altruistic, prosocial and selfish. To facilitate 147 the association of the options with their corresponding function, the position of the stimuli was 148 fixed for each participant but counterbalanced across participants. In experiment 1, the three options were presented simultaneously on the actor's screen. In experiments 2 and 3, two out of 150 the three options were presented. In experiment 2, two-option choice trials (two out of three options) were interspersed with three-option choice trials (as in experiment 1). In the two-option

152 choice trials, the combination of options was randomly assigned across the individuals. In 153 experiment 3 , one of the three possible combinations of two options was constant across the block

154 of trials. Therefore, we ran three different sessions, each one with two options (out of the three) 155 available across trials (Table 1). The monitor of the second chimpanzee showed a mid-grey blank 156 screen throughout the sessions. 
158 Procedure

159 Training and learning phase

160 In the training phase, the individuals were trained to discriminate three different sounds 161 corresponding to the three different outcomes (prosocial, selfish and altruistic). The sounds used 162 in this experiment were unfamiliar to chimpanzees, so they would not associate with the regular 163 rewarding sound used with other touchscreen tasks. In the training phase, the individuals were 164 tested alone. The actor was placed in in front of his/her monitor, and the recipient was separated 165 in the other compartment of the booth. We did not request the actor to touch the screen in this 166 phase. The actor had access to both feeders, including the recipient feeder. The actor could easily 167 hear the sound and pick up the reward on the recipient feeder. This procedure should ensure that 168 the individuals understand that both feeders provided food. We randomly chose which sounds to 169 play paired with the location of the outcome. We ran 200 trials for each individual.

170 In the learning phases, we trained the chimpanzees to associate the assigned shape with their 171 respective function. Both chimpanzees were now placed in the same compartment, in front of their

172 respective monitors (Fig. 1). We ran three sessions, in which only one of the three stimuli was

173 presented for 24 trials: 24 trials with the presentation of the cube, 24 trials with the sphere and 24

174 trials with the cylinder. Shapes' functions were randomly assigned across the subjects.

In the experimental phase, chimpanzees were tested in actor-recipient pairs, in the same compartment of the experimental booth (Fig. 1), approximately $0.40 \mathrm{~m}$ apart. One degree of gaze angle corresponded to approximately $0.7 \mathrm{~cm}$ on the screen at a viewing distance of $40 \mathrm{~cm}$. One 
181 sessions. Each trial was initiated by the actor pressing a green button on the middle bottom of

182 screen. The actor made a choice by touching one of the three options presented on the screen. A

183 food reward (an apple piece, approximately $1 \mathrm{~cm}^{3}$ ) was given according to the assignments of the

184 options and their functions. The three options consisted in rewarding only the actor (selfish (S)),

185 both participants (prosocial $(\mathrm{P})$ ) or only the recipient (altruistic $(\mathrm{A})$ ). Feeder activation was

186 accompanied by two distinctive buzzer sounds with slight temporal delay to indicate clearly which

187 feeder was giving the reward. Throughout the procedure the recipient sat in front of a grey screen.

188 After the completion of the experiment (by the end of the third session) the chimpanzees changed

189 positions: the actor moved to the recipient's place and vice-versa. Each pair received three sessions

190 for each role, totaling six sessions per day.

191 In the experiment 1, we ran three sessions of 48 trials (144 trials in total) for each actor.

192 The actor could choose among three options on the screen: P, S and A (Fig. 2). In experiment 2,

193 option assignments and locations on the screen were as in experiment 1, except that we also

194 reduced the number of options from three to two: prosocial and selfish (P-S), selfish and altruistic

195 (A-S), or prosocial and altruistic (P-A) (Table 1). We ran 32 trials of each combination, giving a 196 total of 96 trials for each subject. These two-option trials were randomly interspersed with three-

197 option trials at a ratio of 1:5, to ensure that chimpanzees could associate this new condition with

198 the previous one, as the conditions have been conducted in different days. However, because our 199 focus was on the two-option trials, we only analysed those trials in this study. By reducing the 200 options in some of the trials, we turned the social event into a more critical decision-making 201 situation than in experiment 1 (three-option-choices) and, hence, increased the social pressure 202 between partners and possible repercussions toward the active partner. 
In experiment 3, to further explore the dynamics of the two-option task and increase the

204

205

206

207

208

209

210

211

212

213

214

215

216

217

218

219

220

221

222

223

224

225 social pressure between the partners, we provided each of the two option choices in blocks of 48 trials in a counterbalanced order across participants (Table 1). Experiment 3 involved presentations of two options at the same time and consisted of 96 trials presented in two sessions for each combination of two trial types: P-S, A-S, P-A.

All experiments were carried out in accordance with the 2002 version of the Guidelines for the Care and Use of Laboratory Primates by the Primate Research Institute, Kyoto University. The experimental protocol was approved by the Animal Welfare and Care Committee of the same institute (protocol\# 2012-090).

Data analysis

Data analysis was performed using R 3.3.1 (R Core Team 2015) in R-studio 0.99.463 (R Studio 2015). For individual testing, we used Chi-square tests for the three-choice experiment (experiment 1) and binomial tests for the two-choice experiment, (experiment 2 and 3), to examine subjects' performance against chance level. We rejected the null hypothesis if $\mathrm{P}<0.05$. Additionally, we use the function geom_smooth, method = "loess" from the package "ggplot2" to fit a line using linear smoothing for the figures corresponding to each experiment. The curve given by geom_smooth function produces an estimate of the conditional mean function. The shaded band represents a pointwise $95 \%$ confidence interval on the fitted values (given by the line).

Results

Experiment 1 
Five out of the six individuals chose the prosocial option above chance level (Chi-squared,

227 Table 2). One of the six individuals (Pn) showed the opposite trend and preferentially choose the

228 selfish option more often than prosocial, and this tendency increased across the trials.

229 Figure 3A shows an increasing overall preference for the prosocial option over all 144 trials

230 for five out of six individuals, while the preference for the selfish and altruistic options decreased

231 over the trials. The selfish individual, Pn was plotted separately (Fig. 3B) to show her preference

232 for the selfish option over the prosocial and altruistic across trials.

233 Experiment 1 reveals an exploratory phase, in which, in the beginning individuals (except

234 for $\mathrm{Cl}$ ) were choosing the three options at similar proportions (first bin of 8 trials, Fig. 3A) until

235 they started showing a preference for the prosocial option with the increase of trials.

237 Experiment 2

238 Four of the six individuals chose the prosocial option above chance level (Fig. 4A). Am 239 did not choose the prosocial option above chance level in this experiment (Binomial test, Table 3).

240 The selfish subject, Pn, kept choosing the selfish option more than the prosocial option, as she did

241 in experiment 1, thereby deviating from the pattern shown by the other participants (Fig. 4B). In

242 this experiment, the proportion of prosocial choices, for three out of the six individuals (Am, Ch

243 and Pn), decreased in this experiment, compared to experiment 1. The probability of mothers

244 choosing the selfish option over the altruistic and prosocial options increased in experiment 2 245 compared to experiment 1 (Fig. 5). Unlike in experiment 1, the chimpanzees' choices did not vary 246 over trials suggesting that individuals may have remembered the symbol assignments from the 247 previous experiment. 
249

250

251

252

253

254

255

256

257

258

259

260

261

262

263

264

265

266

267

268

269

270

Experiment 3

Similar to experiment 1 , the individual's responses showed that five of the six individuals chose the prosocial option above chance level (Binomial test, Table 4). Similar to experiment 2, the proportion of choosing the prosocial key was kept constant across the trials, for five out of six individuals (Fig. 6A). Like in the experiments 1 and 2, Pn stood out from other participants by choosing the selfish option over the prosocial option (Fig. 6B). Overall, the proportion of prosocial choices over selfish increased from the experiment 2 for three individuals ( $\mathrm{Ai}, \mathrm{Am}$ and $\mathrm{Ch}$ ).

Mothers showed a greater tendency than their offspring to choose the selfish option over the altruistic option (Fig. 5A). The probability of choosing the altruistic option also increased in experiment 3 , with the exception of the male offspring (Am), who showed a similar pattern to mothers (Table 4). Similar to experiment 2, an exploratory phase was not observed.

Discussion

Overall, we found that prosocial behavior predominated over selfish and altruistic behaviors (experiment 1). Prosocial responding was slightly more frequent when the alternative was altruistic responding (experiments 2 and 3) compared to selfish, and all individuals show a clear preference for behaving prosocially over the altruistic option. In the early trials of experiment 1, chimpanzees chose among the three options at close to the chance level; however, their options stabilized with increasing experience of the outcome of each choice. In experiments 2 and 3 no such exploratory behavior was observed, suggesting that most of the chimpanzees (with exception of $\mathrm{Cl}$ ) understood and remembered the outcome of their choices from experiment 1 . $\mathrm{Cl}$ always chose the prosocial option from the beginning of experiment 1 and did not explore other outcomes. 
271 Therefore, there is the possibility that she may have just learned that the prosocial symbol was

272 rewarding to her via simple associate learning or she may have learned to avoid the other choices.

Four out of the six chimpanzees showed a tendency towards prosocial behaviour, supporting findings of previous experimental studies (Warneken et al., 2007; Horner et al., 2011a; Melis et al., 2011) and evidence from observations in the wild (Nishida \& Hosaka, 1996; Watts, 1998; Duffy, Wrangham \& Silk, 2007). A potential limitation of the study by Horner et al. (2011a) concerns the low number of repetitions (30 trials). In the present study the proportion of prosocial choices made by the chimpanzees at around 30 trials (i.e. 4 bins) was similar to that in Horner et al. (2011a). However, by increasing the number of trials (by a factor of 4.8) we increased the overall prosocial bias from an average of $60 \%$ in Horner et al. (2011a) to an average of $88 \%$, and to $100 \%$ for five out of six chimpanzees. During the first phase of trials in experiment 1 , chimpanzees chose more equitably among the three options (exploratory phase), before eventually switching their preference for the prosocial option, a preference that persisted until the end of testing. The prosocial-selfish rate found in previous studies (e.g. Silk et al., 2005; Jensen et al., 2006; Horner et al., 2011a) may be, therefore, a consequence of subjects receiving fewer trials. In addition to experiencing more trials in the current experiment, it is also possible that having the two individuals sharing the same compartment during the experiment motivated the chimpanzees to act more prosocially because of fear of repercussions from the partner. However, in the study of Claidière et al (2015) which tested chimpanzee pairs in the same and in separate adjacent enclosures found that chimpanzees behave more prosocially when they were separated. Tennie, Jensen \& Call (2016) have shown that chimpanzees' willingness to help others may depend on the experimental settings, therefore prosociality could arise as a by-product of the 
294 control for the effect of sharing the same chamber in these experiments.

295 In contrast to our results, no modulation of prosocial behavior by relative social rank was

296

297

298

299

300

301

302

303

304

305

306

307

308

309

310

311

312

313

314

315

316 observed in Horner et al. (2011a). It can be argued that the lack of any rank-related influence on prosocial behavior might be due to the physical separation of the two actors in that study. The fear of potential repercussions from the mothers could explain why the female offspring acted more altruistically (given the selfish option) compared to their mothers, in experiment 3.

One may argue that chimpanzees were choosing the prosocial option with the intention of scrounging the reward from the partner, however we did not observe any scrounging behavior or attempt to steal the reward during the experiment. Moreover, we also did not observe any signs of frustration by the partner, when they were most likely to occur, in experiment 3 , when given the choice between acting selfishly or altruistically.

Some previous studies that failed to show, or showed little evidence of prosociality appear more complex methodologically and may have require extra cognitive effort compared to the task used in our study. Examples include using tokens to exchange for food rewards with a human experimenter (e,g. Horner et al., 2011a), or using a stick as a tool to dislodge food rewards (e.g. Vonk et al., 2008). In those cases, actors behaved "prosocially" even in a ghost condition in which no conspecific was present. Given the settings of our experiment, we could not run a condition with the partner being absent. If we had run the ghost condition, the actor could try to maximize the reward by choosing the prosocial option in the absence of a partner, thus spoiling the association between the key and the reward outcome. If we had blocked the passage of the recipient, we would have to run the experiment with both subjects separated from the beginning which was not our goal, as we wanted to test individuals in the same compartment to increase social pressure. Therefore, to be able to run a ghost condition we would have to change our settings 
317 from the start. Further experiments should take these matters into account.

318 Although rank turned out to be an important factor in our study (with mothers being the 319 more dominant individuals), because we tested only mother-offspring pairs, we could not examine

320 the influence of kinship separate from rank. Considering the various differences we found in the

321 response patterns between the mothers and their respective offspring, we cannot support the

322 suggestion that chimpanzees return past favors (Gomes, Mundry \& Boesch, 2009; Gomes \&

323 Boesch, 2011). As stated in Horner et al. (2011a), this lack of evidence might be related to the fact

324 that cooperative behaviors such as hunting (Boesch \& Boesch, 1989; Boesch, 1994) patrolling and

325 coalitions (Mitani, Merriwether \& Zhang, 2000) are more typical of male than female

326 chimpanzees. We tested five females and only one male; clearly, further studies are needed to

327 address the question of sex differences regarding prosocial tendencies.

One chimpanzee, Pn, showed a preference for the selfish option over altruistic and prosocial options, and this tendency was maintained across experiments. Pn's behavior in combination with that of the two mothers from the other two pairs led to an overall increase in the proportion of selfish vs. prosocial options. However, it should be noted that not all mothers showed higher proportions than their offspring, also reflected in the greater dissimilarity among individuals in P-S than P-A trials. Pn chose selfish when selfish was an option, and prosocial when selfish was not an option; she never chose the altruistic option. In a previous study, the same individual failed to help a partner in the absence of any request, while all other individuals tested did so (Yamamoto, Humle \& Tanaka, 2012). There is one clear difference in the life history of Pn compared to other participants: Pn was hand-raised by humans. If food is always provided by human caretakers, there is no dependence on other chimpanzees, hence sharing food or begging for food from other chimpanzees may be unnecessary. Previous studies showed the opposite pattern, however when 
340 having a human as mediator (Warneken \& Tomasello, 2006). Given our small sample size this

341 explanation is speculative. However, it raises the interesting possibility that the tendencies to share

342 food (prosocial) or provide food to other (altruistic) are not genetically predetermined behavioral

343 traits; instead, they could arise from a gene-environment interaction (Plomin, DeFries \& Loehlin,

344 1977). Further studies are required to examine the effect of chimpanzee rearing history on

345 prosocial and altruistic tendencies. One offspring participant showed an increasing trend toward

346 choosing selfish over altruistic options (Am). This individual was an 11-year-old male who at the

347 time of the study was involved in competition with the alpha-male of the group. This social

348 circumstance might indicate a switching point for Am from offspring behavior to more adult-like

349 behavior.

In summary, while it is valid to question (Skoyles, 2011) a 60\% advantage for prosocial

351

352

353

354

355

356

357

358

359

360

361

above selfish options (Horner et al., 2011a), we found prosocial responses at much higher rates with increasing task experience. This factor could explain the differences found in Horner et al. (2011a). Sampling alternative options to confirm the game's contingencies (Horner et al., 2011a) did not occur. Notwithstanding the small sample size, based on our results we suggest that the rank-relationship between partners, in contrast to Horner et al. (2011a), and supporting other authors (Melis, Schneider \& Tomasello, 2011; Yamamoto, Humle \& Tanaka, 2012) may modulate prosocial tendencies: with increasing social pressure and hence fear of repercussions from their mothers, female offspring showed altruistic behavior.

Overall, this study confirms that chimpanzees are not "indifferent to the welfare of others" (Silk et al., 2005), however their choices reveal a balanced interplay of rationally maximizing their own gains (Jensen, Call \& Tomasello, 2007) while circumventing repercussions from the 
362 partner (de Waal, 1982). Further, we provide a new framework for examining social cognition in

363 a computer-guided testing procedure, allowing better identification of effect-modulating factors.

Conclusion

We provide a new framework for accessing prosociality in non-human primates, through the utilization of a controlled computer apparatus. This improvement of the old paradigm allows us to increase the number of trials and prevents the direct participation of humans in the task that could be a distracter or bias in the chimpanzees' choices. Additionally, the touchscreen methodology developed in the study helps control for the effect of visible food along with the ability to increase trial numbers (Cronin 2012). when they are faced with two other options: being selfish or altruistic by benefiting themselves or the other, respectively. Ultimately, we hypothesize that the rearing history of chimpanzees and the rank-relationship between partners influenced their positive or negative response towards prosociality.

We thank Dr. James R. Anderson for his comments on the earlier versions of this manuscript, Dr. Masaki Tomonaga and the staff of the Language and Intelligence Section for their help and useful comments. We kindly thank the Center for Human Evolution Modelling Research at the Primate Research Institute for daily care of the chimpanzees. We also appreciate the valuable 
383 input by Dr. Lydia Hopper and three anonymous reviewers.

384

385

386

387

388

389

390

391

392

393

394

395

396

397

398

399

400

401

402

References

Adachi I. 2014. Spontaneous Spatial Mapping of Learned Sequence in Chimpanzees: Evidence for a SNARC-Like Effect. PLoS ONE 9:e90373. doi: 10.1371/journal.pone.0090373.

Batson CD, Powell AA. 2003. Altruism and Prosocial Behavior. In: Weiner IB, Freedheim DK, Schinka JA, Velicer WF, eds. Handbook of psychology. Wiley, New York: Wiley, 282316.

Boesch C .1994. Cooperative hunting in wild chimpanzees. Animal Behaviour 48:653-667. doi: 10.1006/anbe.1994.1285.

Boesch C, Boesch H. 1989. Hunting behavior of wild chimpanzees in the Taï National Park. American Journal Physical Anthropology 78:547-573. doi: 10.1002/ajpa.1330780410.

Claidière N, Whiten A, Mareno MC, Messer EJE, Brosnan SF, Hopper LM, Lambeth SP, Schapiro SJ, McGuigan N. 2015. Selective and contagious prosocial resource donation in capuchin monkeys, chimpanzees and humans. Scientific Reports 5:7631. doi: 10.1038/srep07631.

Crockford C, Wittig RM, Mundry R, Zuberbühler K. 2012. Wild Chimpanzees Inform Ignorant Group Members of Danger. Current Biology 22:142-146. doi: 10.1016/j.cub.2011.11.053.

Cronin KA, Schroeder KKE, Snowdon CT. 2010. Prosocial behaviour emerges independent of reciprocity in cottontop tamarins. Proceedings of the Royal Society of London. Series B, Biological Sciences. doi: 10.1098/rspb.2010.0879. 
403 de Waal F. 1989. Chimpanzee politics: Power and sex among apes. Baltimore: Johns Hopkins $404 \quad$ University Press.

405

406

407

408

409

410

411

412

413

414

415

416

417

418

419

420

421

de Waal FBM. 2008. Putting the Altruism Back into Altruism: The Evolution of Empathy. Annual Review of Psychology 59:279-300. doi: 10.1146/annurev.psych.59.103006.093625.

Duffy KG, Wrangham RW, Silk JB. 2007. Male chimpanzees exchange political support for mating opportunities. Current Biology 17:R586-R587.

Fehr E, Fischbacher U. 2003. The nature of human altruism. Nature 425:785-791.

Fehr E, Fischbacher U. 2004. Third-party punishment and social norms. Evolution and Human Behavior 25:63-87. doi: 10.1016/S1090-5138(04)00005-4.

Fehr E, Gächter S. 2002. Altruistic punishment in humans. Nature 415:137-140.

Gomes CM, Boesch C. 2011. Reciprocity and trades in wild West African chimpanzees. Behavioral Ecology and Sociobiology 65:2183-2196. doi: 10.1007/s00265-011-1227-x.

Gomes CM, Mundry R, Boesch C. 2009. Long-term reciprocation of grooming in wild West African chimpanzees. Proceedings of the Royal Society of London. Series B, Biological Sciences 276:699-706. doi: 10.1098/rspb.2008.1324.

Hare B, Call J, Tomasello M. 2001. Do chimpanzees know what conspecifics know? Animal Behaviour 61:139-11. doi: 10.1006/anbe.2000.1518.

Hirata S. 2009. Chimpanzee social intelligence: selfishness, altruism, and the mother-infant bond. Primates 50:3-11. doi: 10.1007/s10329-008-0122-1. 
422 Hoffman ML. 1978. Psychological and Biological Perspectives on Altruism. International Journal 423 of Behavioral Development 1:323-339. doi: 10.1177/016502547800100403.

424

425

426

427

428

429

430

431

432

433

434

435

436

437

438

439

440

441

Horner V, Carter JD, Suchak M, de Waal FBM. 2011a. Spontaneous prosocial choice by chimpanzees. Proceedings of the National Academy of Sciences 108:13847-13851. doi: 10.1073/pnas.1111088108.

Horner V, Carter JD, Suchak M, de Waal FB. 2011b. Reply to Skoyles: Misplaced assumptions of perfect human prosociality. Proceedings of the National Academy of Sciences 108:E836E836.

House BR, Silk JB, Lambeth SP, Schapiro SJ. 2014. Task design influences prosociality in captive chimpanzees (Pan troglodytes). PLOS ONE 9:e103422.

Jensen K, Call J, Tomasello M. 2007. Chimpanzees Are Rational Maximizers in an Ultimatum Game. Science 318:107-109. doi: 10.1126/science.1145850.

Jensen K, Hare B, Call J, Tomasello M. 2006. What's in it for me? Self-regard precludes altruism and spite in chimpanzees. Proceedings of the Royal Society B: Biological Sciences 273:1013-1021. doi: 10.1098/rspb.2005.3417.

Kim Y, Martinez L, Choe JC, Lee DJ, Tomonaga M. 2015. Orangutans (Pongo spp.) do not spontaneously share benefits with familiar conspecifics in a choice paradigm. Primates 56:193-200. doi: 10.1007/s10329-015-0460-8.

Lakshminarayanan VR, Santos LR. 2008. Capuchin monkeys are sensitive to others' welfare. Current Biology 18:R999-R1000. doi: 10.1016/j.cub.2008.08.057. 
442 Langergraber KE, Mitani JC, Vigilant L. 2007. The limited impact of kinship on cooperation in 443 wild chimpanzees. Proceedings of the National Academy of Sciences 104:7786-7790.

444 Martin, CF, R Bhui, P Bossaerts, T Matsuzawa, and C Camerer. 2014. Chimpanzee choice rates 445 in competitive games match equilibrium game theory predictions. Scientific Reports doi: 446 $10.1038 /$ srep05182.

447

448

449

450 451

452 453

454

455

456

457

458

459 sample tasks: public information use in a fully automated testing environment. Animal Cognition 14:893-902. doi: 10.1007/s10071-011-0424-3.

Matsuzawa T. 2003. The Ai project: historical and ecological contexts. Animal Cognition 6:199211.

Matsuzawa T. 2006. Sociocognitive development in chimpanzees: a synthesis of laboratory work and Fieldwork. In: Matsuzawa T, Tomonaga M, Tanaka M, eds. Cognitive development in chimpanzees. Tokyo:Springer, pp 3-33.

McGrew WC. 2010. In search of the last common ancestor: New findings on wild chimpanzees. Philosophical Transactions of the Royal Society B 365: 3267-3276.

Melis AP, Schneider A-C, Tomasello M. 2011. Chimpanzees, Pan troglodytes, share food in the same way after collaborative and individual food acquisition. Animal Behaviour. doi: 10.1016/j.anbehav.2011.05.024. 
460 Melis AP, Warneken F, Jensen K, Schneider A-C, Call J, Tomasello M. 2011. Chimpanzees help

461

462

463

464

465

466

467

468

469

470

471

472

473

474

475

476

477

478

479 conspecifics obtain food and non-food items. Proceedings of the Royal Society B: Biological Sciences 278:1405-1413. doi: 10.1098/rspb.2010.1735.

Mitani JC, Merriwether DA, Zhang C. 2000. Male affiliation, cooperation and kinship in wild chimpanzees. Animal Behaviour 59:885-893. doi: 10.1006/anbe.1999.1389.

Mueller DC. 1986. Rational egoism versus adaptive egoism as fundamental postulate for a descriptive theory of human behavior. Public Choice 51:3-23. doi: 10.1007/BF00141682.

Nishida T, Hosaka, K. 1996. Coalition strategies among adult male chimpanzees of the Mahale Mountains, Tanzania. In: McGrew W, Marchant L, Nishida T, eds. Great Ape Societies. Cambridge: Cambridge University Press, 114-134.

Plomin R, DeFries JC, Loehlin JC. 1977. Genotype-environment interaction and correlation in the analysis of human behavior. Psychological Bulletin 84:309-322.

Silk JB, Brosnan SF, Vonk J, Henrich J, Povinelli D J, Richardson AS, Lambeth SP, Mascaro J, Shapiro SJ. 2005. Chimpanzees are indifferent to the welfare of unrelated group members. Nature 437:1357-1359. doi: 10.1038/nature04243.

Skerry AE, Sheskin M, Santos LR. 2011. Capuchin monkeys are not prosocial in an instrumental helping task. Animal Cognition 14:647-654. doi: 10.1007/s10071-011-0399-0.

Skoyles JR. 2011. Chimpanzees make mean-spirited, not prosocial, choices. Proceedings of the National Academy of Sciences 108:E835-E835. 
480 Suchak M, Epple, TM, Campbell MW, de Waal FBM. 2014. Ape duos and trios: spontaneous 481 cooperation with free partner choice in chimpanzees. PeerJ 2:e417

482

483

484

485

486

487

488

489

490

491

492

493

494

495

496

497

498

Suchak M, de Waal FBM. 2012. Monkeys benefit from reciprocity without the cognitive burden. Proceedings of the National Academy of Sciences 109:15191-15196. doi: 10.1073/pnas.1213173109.

Takimoto A, Fujita K. 2011. I acknowledge your help: capuchin monkeys' sensitivity to others’ labor. Animal Cognition 14:715-725. doi: 10.1007/s10071-011-0406-5.

Tennie C, Jensen K, Call J. 2016. The nature of prosociality in chimpanzees. Nature Communications 7:13915.

Vonk J, Brosnan SF, Silk JB, Henrich J, Richerdson AS, Lambeth SP, Schapiro SJ, Povinelli DJ. 2008. Chimpanzees do not take advantage of very low cost opportunities to deliver food to unrelated group members. Animal Behaviour 75:1757-1770. doi: 10.1016/j.anbehav.2007.09.036.

Warneken F, Tomasello M. 2006. Altruistic Helping in Human Infants and Young Chimpanzees. Science 311:1301-1303. doi: 10.1126/science.1121448.

Warneken F, Hare B, Melis AP, Hanus D, Tomasello M. 2007. Spontaneous altruism by chimpanzees and young children. PLoS Biology 5:e184.

Warneken F, Tomasello M. 2009. The roots of human altruism. British Journal of Psychology 100:455-471. doi: 10.1348/000712608X379061. 
499 Watts DP. 1998. Coalitionary mate guarding by male chimpanzees at Ngogo, Kibale National $500 \quad$ Park, Uganda. Behavioral Ecology and Sociobiology 44:43-55.

501 Yamamoto S, Humle T, Tanaka M. 2012. Chimpanzees' flexible targeted helping based on an 502 understanding of conspecifics' goals. Proceedings of the National Academy of Sciences $503 \quad 109: 3588-3592$. doi: 10.1073/pnas.1108517109.

504

505

506 


\section{Figure 1}

Pan (Pn) and Pal (PI) performing the task and sharing the same compartment. Pan as the actor on the right and Pal as the recipient on the left. Photo credit: Renata Mendonça.

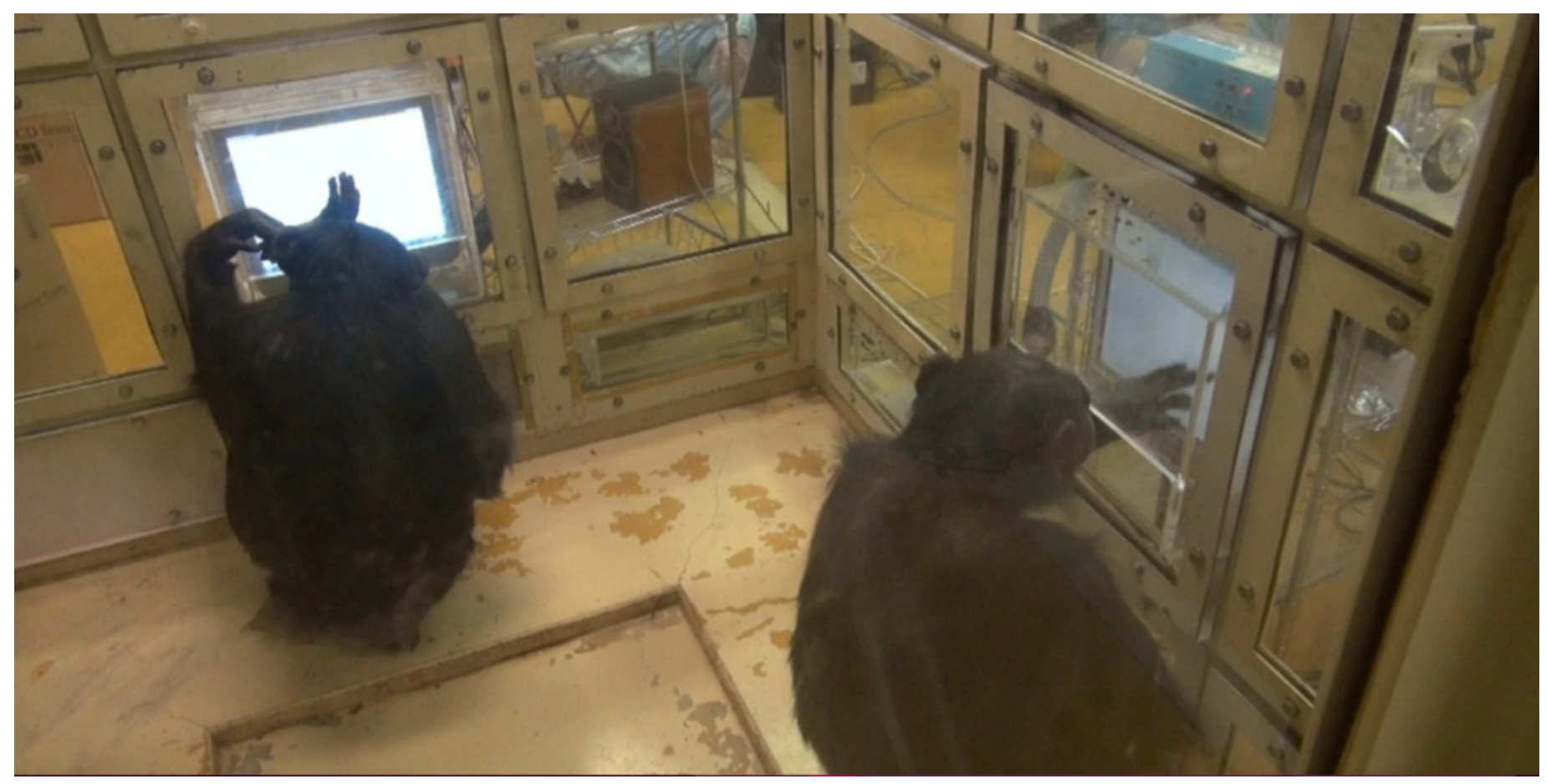


Figure 2

Procedure of the experiment 1 "three choices condition". Grey screen represents the screen of the actor. 
3) Choose one of the 3 options

1) Press the circular button to initiate the task

2) The 3 options are
shown on the screen

2) The 3 options are
shown on the screen

Actor's screen
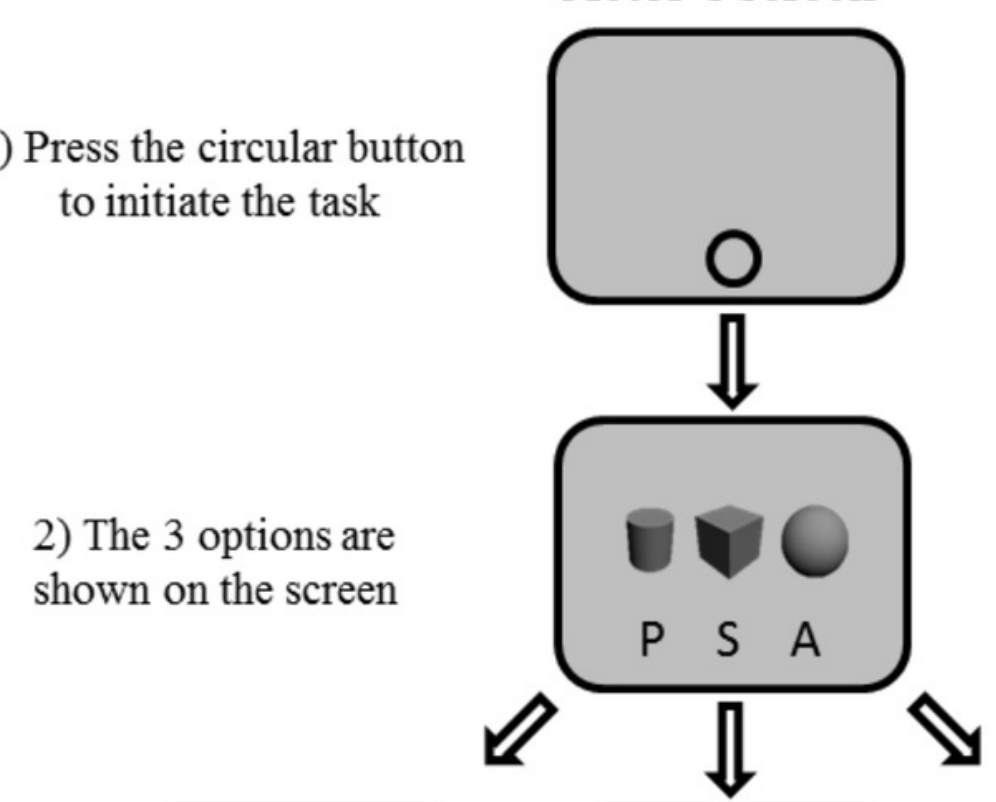

4) Choice's outcome
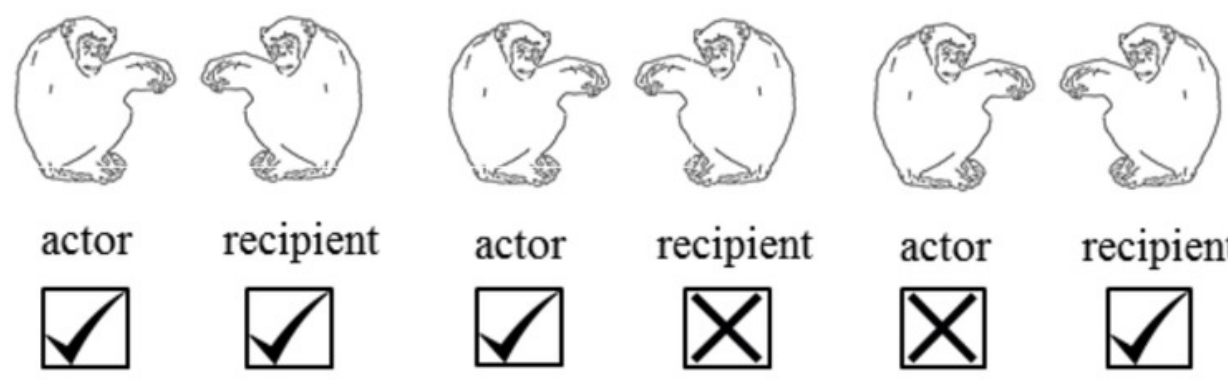

actor

recipient
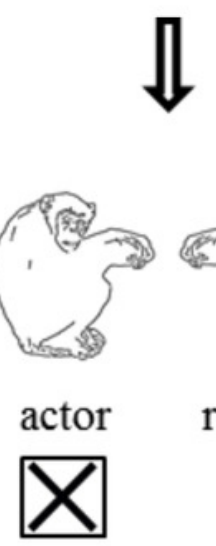

A

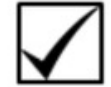

$\square$ Individual rewarded

囚 Individual not rewarded 
Figure 3

Proportion of the cumulative mean in the experiment 1 for selfish, altruistic and prosocial options as a function of trials (X-axis) for five individuals (A) and Pan (B).

Trials are grouped in bins (each comprising 8 trials) for a total of 144 trials, i.e. 18 bins. Error bars represent standard errors of the means. The shaded band represents the pointwise 95\% confidence interval on the fitted value.

A

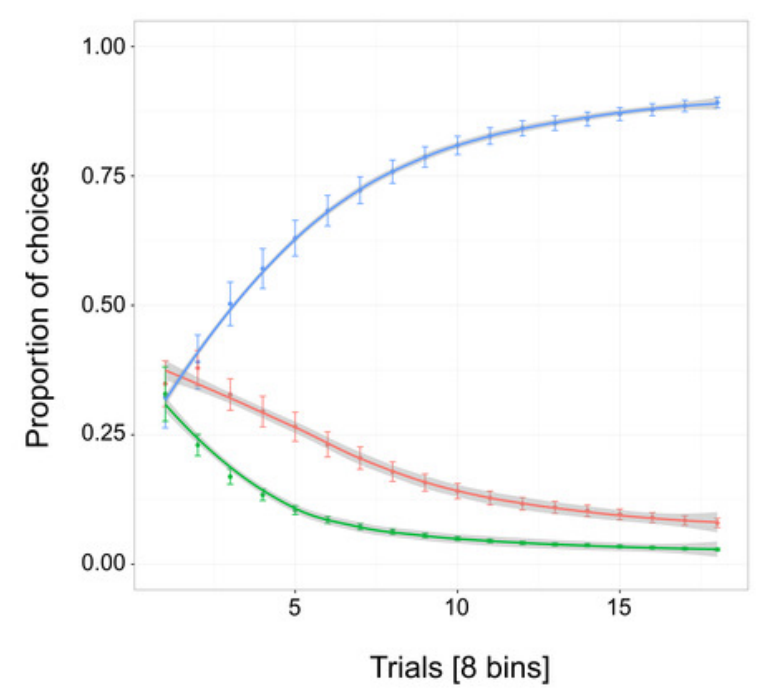

B

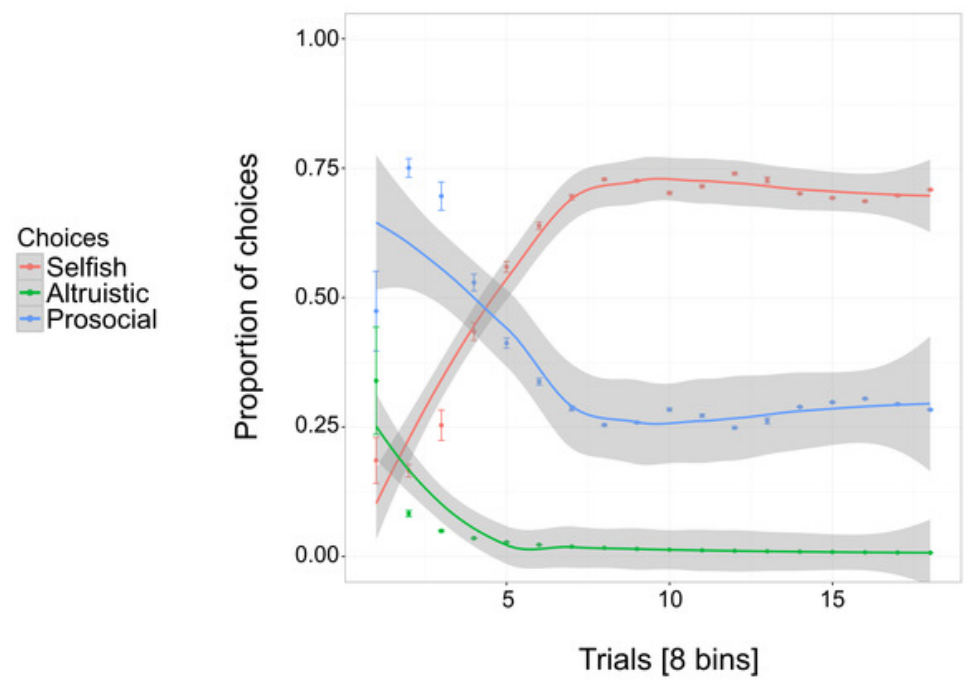




\section{Figure 4}

Proportion of the cumulative mean for combinations of two options: S-A, (selfish and altruistic), P-A (prosocial and altruistic) and P-S (prosocial and selfish) in experiment 2 for five individuals (A) and Pan (B).

"Altruistic (A-S)" means choosing the altruistic option when the altruistic and the selfish options are given; "Prosocial (P-A)" means choosing the prosocial option when the prosocial and the altruistic choices are given; "Prosocial (P-S)" means choosing the prosocial option when the prosocial and the selfish options are given. Error bars represent standard errors of the means $(A)$. The shaded band represents the pointwise $95 \%$ confidence interval on the fitted value.

A

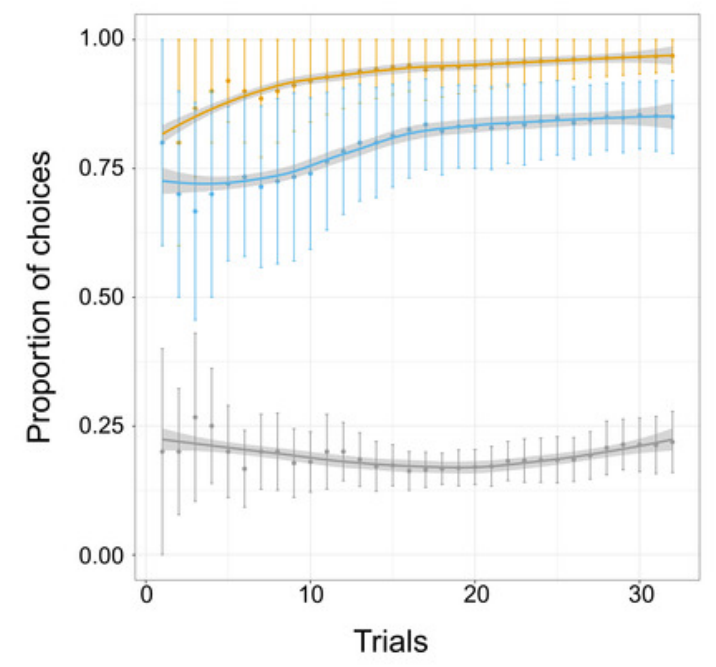

B

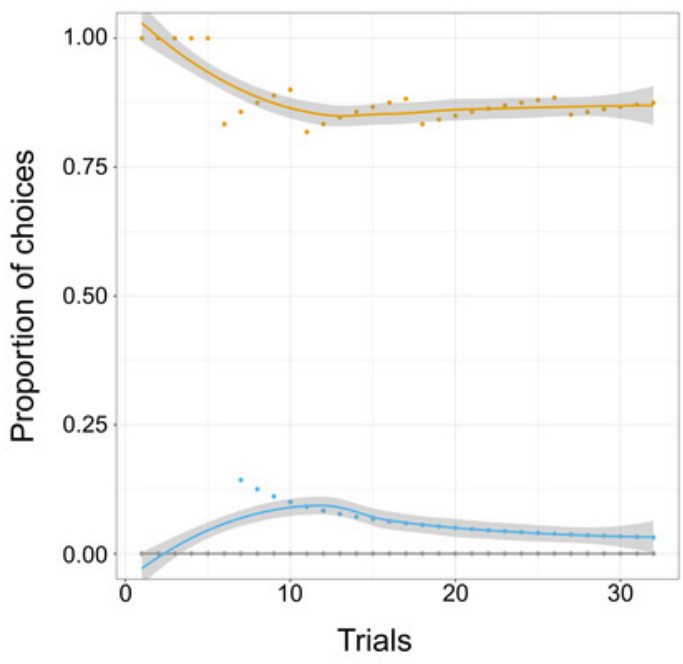




\section{Figure 5}

Mean proportion of choices for S-A (selfish and altruistic) trials $(A)$, and mean proportion of choices for S-P (selfish and prosocial) trials (B) split into offspring and mothers for experiment 2 and 3.

"Selsfish (A-S)" means choosing the selfish option when the altruistic and the selfish options are given (A); "Selfish (P-S)" means choosing the selfish option when the prosocial and the selfish choices are given (B). Error bars are standard errors of the means.

A

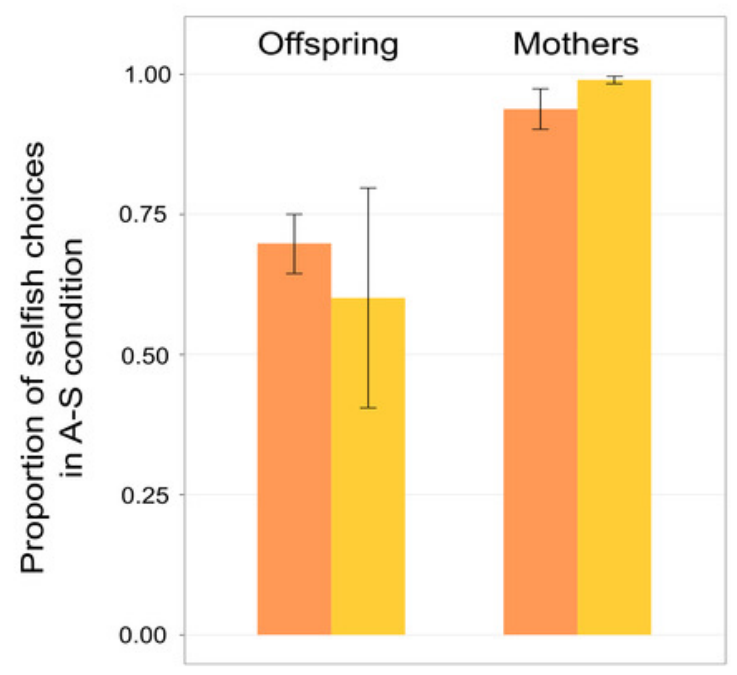

B

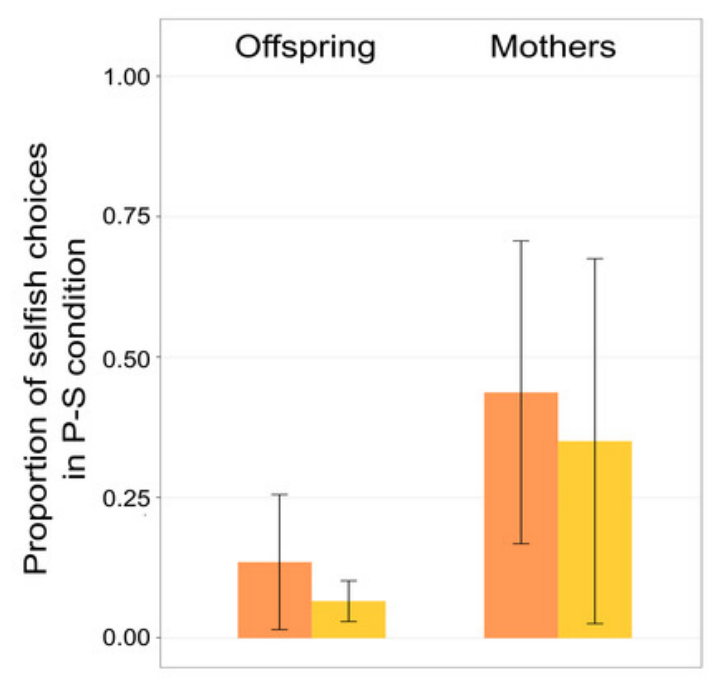

Experiments

\#2 \#3 


\section{Figure 6}

Proportion of the cumulative mean for combinations of two options: S-A, (selfish and altruistic), P-A (prosocial and altruistic) and P-S (prosocial and selfish) in experiment 3 for five individuals $(A)$ and Pan (B).

"Altruistic (A-S)" means choosing the altruistic option when the altruistic and the selfish options are given; "Prosocial (P-A)" means choosing the prosocial option when the prosocial and the altruistic choices are given; "Prosocial (P-S)" means choosing the prosocial option when the prosocial and the selfish options are given. Trials are grouped in bins (each comprising 6 trials) for a total of 96 trials, i.e. 16 bins. Error bars represent standard errors of the means. The shaded band represents the pointwise 95\% confidence interval on the fitted value.
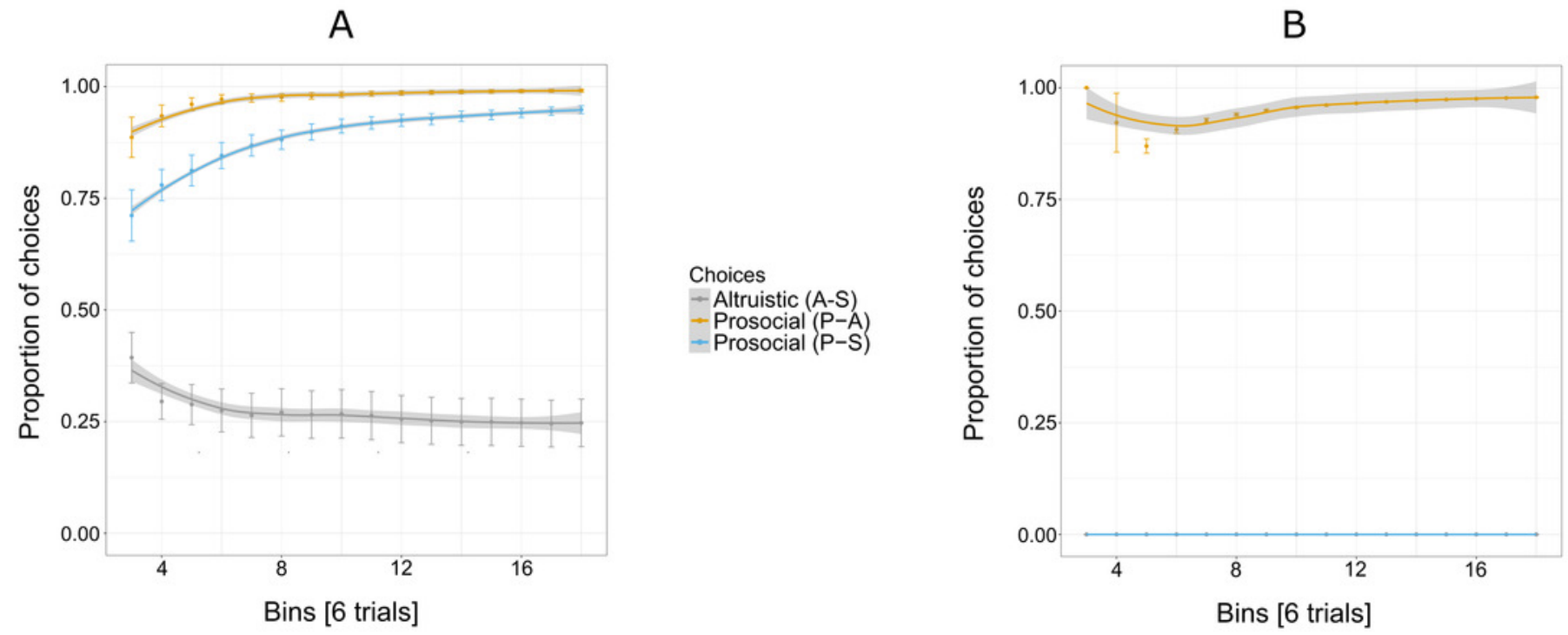


\section{Table $\mathbf{1}$ (on next page)}

Summary of the description of each experiment. 


\begin{tabular}{|c|c|}
\hline Experimental condition & Description of the task \\
\hline Experiment 1 & $\begin{array}{l}3 \text { choices were presented - prosocial (P), selfish (S), } \\
\text { altruistic (A) for } 3 \text { sessions of } 48 \text { trials, totalling } 144 \\
\text { trials (Figure } 2 \text { ). }\end{array}$ \\
\hline Experiment 2 & $\begin{array}{l}2 \text { out of } 3 \text { choices }(\mathrm{P}-\mathrm{S}, \mathrm{S}-\mathrm{A}, \mathrm{P}-\mathrm{A}) \text { were presented } \\
\text { randomly, interspersed with } 3 \text { choice trials }(\mathrm{P}, \mathrm{S}, \mathrm{A}) \text { at } \\
\text { a rate of } 1: 5 \text {. We ran } 3 \text { sessions of } 32 \text { trials. }\end{array}$ \\
\hline Experiment 3 & $\begin{array}{l}2 \text { out of } 3 \text { choices were presented. We ran } 96 \text { trials for } \\
\text { each pair of choices (P-S, S-A, P-A). Each pair was } \\
\text { presented in a block of } 48 \text { trials and the order of choices } \\
\text { counterbalanced between individuals. }\end{array}$ \\
\hline
\end{tabular}




\section{Table 2(on next page)}

Results of the chi-square test for all the individuals in the experiment 1. 
1

2

\begin{tabular}{ccccccc}
\hline Individuals & Age class & $\begin{array}{c}\text { Prosocial } \\
\text { choice }\end{array}$ & $\begin{array}{c}\text { Selfish } \\
\text { choice }\end{array}$ & $\begin{array}{c}\text { Altruistic } \\
\text { choice }\end{array}$ & Chi-square & P-value \\
\hline $\mathrm{Ai}$ & Mother & 0.82 & 0.15 & 0.03 & 155.79 & $<0.001$ \\
$\mathrm{Am}$ & Offspring & 0.84 & 0.13 & 0.03 & 168.29 & $<0.001$ \\
$\mathrm{Ch}$ & Mother & 0.91 & 0.05 & 0.03 & 215.38 & $<0.001$ \\
$\mathrm{Cl}$ & Offspring & 1 & 0 & 0 & 288 & $<0.001$ \\
$\mathrm{Pn}$ & Mother & 0.29 & 0.70 & 0.01 & 105.29 & $<0.001$ \\
$\mathrm{Pl}$ & Offspring & 0.9 & 0.06 & 0.03 & 210.29 & $<0.001$ \\
\hline
\end{tabular}




\section{Table 3 (on next page)}

Results of the binomial test for all the individuals in the experiment 2 . 
1

\begin{tabular}{lrrrrrrr}
\hline Individuals & Age class & \multicolumn{1}{c}{$\begin{array}{c}\text { Prosocial } \\
\text { choice over } \\
\text { selfish }\end{array}$} & P-value & $\begin{array}{c}\text { Prosocial } \\
\text { choice over } \\
\text { altruistic }\end{array}$ & $\begin{array}{c}\text { P-value } \\
\text { choice over } \\
\text { altruistic }\end{array}$ & $\begin{array}{c}\text { Selfish } \\
\text { P-value }\end{array}$ \\
\hline $\mathrm{Ai}$ & Mother & 0.90 & $<0.001$ & 1 & $<0.001$ & 0.94 & $<0.001$ \\
$\mathrm{Am}$ & Offspring & 0.62 & 0.21 & 1 & $<0.001$ & 0.75 & 0.007 \\
$\mathrm{Ch}$ & Mother & 0.75 & 0.007 & 0.84 & $<0.001$ & 0.88 & $<0.001$ \\
$\mathrm{Cl}$ & Offspring & 1 & $<0.001$ & 1 & $<0.001$ & 0.50 & 0.378 \\
$\mathrm{Pn}$ & Mother & 0.03 & $<0.001$ & 0.88 & $<0.001$ & 1 & $<0.001$ \\
$\mathrm{Pl}$ & Offspring & 0.97 & $<0.001$ & 1 & $<0.001$ & 0.75 & 0.007 \\
\hline
\end{tabular}




\section{Table 4(on next page)}

Results of the binomial test for all the individuals in the experiment 3. 
1

2

\begin{tabular}{lrrrrrrr}
\hline Individuals & Age class & \multicolumn{2}{c}{$\begin{array}{c}\text { Prosocial } \\
\text { choice over } \\
\text { selfish }\end{array}$} & P-value & $\begin{array}{c}\text { Prosocial } \\
\text { choice over } \\
\text { altruistic }\end{array}$ & $\begin{array}{c}\text { P-value } \\
\text { choice over } \\
\text { altruistic }\end{array}$ & $\begin{array}{c}\text { Pelfish } \\
\text { Pi }\end{array}$ \\
\hline $\mathrm{Ai}$ & 0.99 & $<0.001$ & 1 & $<0.001$ & 0.99 & $<0.001$ \\
$\mathrm{Am}$ & Mother & 0.93 & $<0.001$ & 1 & $<0.001$ & 0.98 & $<0.001$ \\
$\mathrm{Ch}$ & Offspring & 0.96 & $<0.001$ & 1 & $<0.001$ & 0.98 & $<0.001$ \\
$\mathrm{Cl}$ & Offspring & 1 & $<0.001$ & 1 & $<0.001$ & 0.32 & $<0.001$ \\
$\mathrm{Pn}$ & Mother & 0.02 & $<0.001$ & 0.98 & $<0.001$ & 1 & 0.5 \\
$\mathrm{Pl}$ & Offspring & 0.88 & $<0.001$ & 0.96 & $<0.001$ & 0.5 & 1 \\
\hline
\end{tabular}

3 